\title{
Rapid Detection of Difficult-to-Culture Bacterial Pathogens Using Real-time Nanopore Sequencing
}

\author{
Aycan Gündoğdu¹,2, Ayşegül Ulu-Kılıç3, Hüseyin Kılıç', O. Ufuk Nalbantoğlu2,4 \\ 1 Department of Microbiology and Clinical Microbiology, Erciyes University School of Medicine, Kayseri, Turkey \\ 2 Cenome and Stem Cell Center (GenKok), Erciyes University, Kayseri, Turkey \\ 3 Department of Infectious Diseases and Clinical Microbiology, Erciyes University School of Medicine, Kayseri, Turkey \\ 4 Department of Computer Engineering, Engineering Faculty, Erciyes University, Kayseri, Turkey
}

\begin{abstract}
Objective: Timely diagnosis is crucial for successful treatment of infections. Contemporary diagnostics of clinically significant infections relies on a variety of laboratory-based tests. However, the routine microbiological methods, per se are not always confirmatory for the accurate diagnosis in some cases with a significant time delay. Rapid detection of causative agents using nanopore-based metagenomic sequencing is reported in a case of suspected tuberculosis not confirmed using conventional microbiology diagnostics, and in a case of suspected brucellosis with delayed confirmation by conventional microbiology.

Materials and Methods: This report includes two cases. Patient-1 was clinically diagnosed with peritoneal tuberculosis while Patient-2 had relapsing brucellosis. Cultures and PCR were negative for the peritoneal fluid of Patient-1. Serological determinants for brucellosis were under the thresholds for Patient-2. The blood culture was positive for Brucella spp; however, the results were available only after seven days. Peritoneal fluid and blood sample were obtained from Patient-1 and Patient-2, respectively. Total DNA was extracted using commercial kits. Shotgun metagenomic sequencing was performed using Oxford Nanopore Technologies. Read-event data were base-called by the software Metrichor using workflow v2.39.3.

Results: For Patient-1, the first Mycobacterium tuberculosis reads were confidently detected in 20 minutes, for Patient-2 the first Brucella melitensis read was detected at 30-minutes-timepoint. Overall, the pathogens were detected within 6 hours. We demonstrated a real-time nanopore sequencing technology for the rapid detection of infectious agents in two crucial cases where traditional microbiological methods failed to reveal the pathogens.

Conclusion: In this report, we present that real-time sequencing can not only detect conventionally unrevealed pathogens, but it can achieve this in a tight time-frame. Therefore, it could be said that the nanopore sequencing has the promise to enhance our ability to diagnose infectious diseases.

Keywords: Rapid pathogen detection, Peritoneal tuberculosis, Brucellosis, Real-time nanopore sequencing, MinION (Oxford Nanopore)
\end{abstract}

Corresponding Author: Aycan Gündoğdu

E-mail: agundogdu@erciyes.edu.tr

Received: July 9, 2019 Accepted: December 3, 2019 Published: December 25, 2019

\section{Suggested citation:}

Gündoğdu A, Ulu-Kılıç A, Kılış H, Nalbantoğlu OU. Rapid detection of difficult-to-culture bacterial pathogens using real-time nanopore sequencing. Infect Dis Clin Microbiol 2019; 3: 128-133.

DOI: 10.36519/idcm.2019.19015 


\section{INTRODUCTION}

I nfectious diseases are the leading causes of mortality and morbidity worldwide (1). Current diagnosis of clinically significant infectious agents is based on a variety of laboratory-based tests including microscopy, culture, immunoassays and nucleic acid amplification. The majority of these techniques relies on conventional culture-based profiling of clinical samples (2). However, culturing is typically a time-resourceful operation with turnaround durations of a few days, and even weeks for cultures which exhibit slower growth (3). The culture-based diagnosis is also not plausible in the situations that unculturable microbes are the culprits as well as in the cases that the sampling is conducted after initiating the antibiotic treatment. Due to these certain disadvantages of the culture-based convention, assisting technologies such as MALDI-TOF, PCR, hybridization-microarrays etc. are employed to achieve rapid turnaround performances and higher accuracies in clinical pathogen detection (4-5). These technologies provide rapid detection with relatively higher accuracy; however, they are only applicable to predetermined pathogens. Thus, there is a significant concern about their sensitivity. The need for accurate detection for both known and emerging pathogens has been possible by the advancements in next-generation DNA sequencing (6). Thanks to metagenomic strategies and bioinformatics analyses, novel diagnostic tools for the detection of pathogens is available (7-8). However, while offering plausibly unbiased diagnosis, the second generations sequencing, which is the currently used in routine diagnostics, has several disadvantages such as long sequencing durations, thus unfavorable sample-to-answer turnaround times, infeasibility of single sample usage, and unavailability of on-site usage (e.g. out-of-lab availability in the field in cases of outbreaks). Therefore, there is a need to accelerate the diagnosis of microbiological analyses.

The third generation (real-time) sequencing technology is a favourable candidate to address the challenges mentioned above. This work aimed to apply real-time nanopore sequencing-based confirmation of clinically significant yet laboratory-confirmation-negative peritoneal tuberculosis (TB) and a case of brucellosis with delayed culture results.

\section{MATERIALS AND METHODS}

Two hospitalized patients who were clinically diagnosed with peritoneal tuberculosis and brucellosis were included in this work.

Patient-1: A 62-year-old female patient was referred to our clinic with the symptoms of long-standing abdominal distension and fever. At the first examination, her body temperature was $39^{\circ} \mathrm{C}$ and her blood pressure was 120/80 $\mathrm{mmHg}$, and pulse rate was $88 / \mathrm{min}$. Her cardiac and respiratory examination was normal. We did not observe organomegaly but determined massive ascites on abdominal percussion. In laboratory analysis; her complete blood count and biochemical parameters were as follows: WBC: $4.75 \times 103 / \mu \mathrm{L}(4.8-10.7$

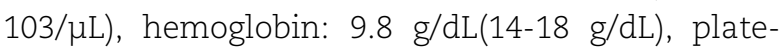

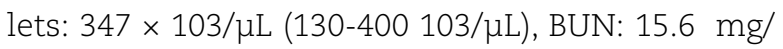
$\mathrm{dL}(6-20 \mathrm{mg} / \mathrm{dL})$, creatinine: $0.78 \mathrm{mg} / \mathrm{dL}(0.7-1.2 \mathrm{mg} /$ dL), AST: $18 \mathrm{U} / \mathrm{L}$ (0-40 U/L), ALT:14 U/L (0-41 U/L), ALP:72 U/L(40-130 U/L), GGT:14 U/L(10-71 U/L) and LDH:226 U/L(135-225 U/L). Her C-reactive protein level was 96 mg/L (0-6 mg/L).

Abdominal CT scan revealed increased peritoneal thickness, linear infiltrative lines in the mesenteric fatty tissue and extensive free abdominal fluid (these signs were interpreted as compatible with peritonitis carcinomatosis or tuberculosis). Peritoneal biopsy revealed chronic granulomatous inflammation composed with giant cells. The patient had TST (tuberculosis skin test) size of $18 \mathrm{~mm}$.

\section{HIGHLIGHTS}

- Pathogens in two different cases (peritoneal tuberculosis and brucellosis) were detected within 6 hours after sampling.

- Shotgun metagenomic sequencing of clinical samples was experienced to be a feasible methodology to be deployed in rapid infectious agent detection for the cases considered.

- The novel real-time nanopore sequencing technology (3rd generation) enables observing the results within hours, opening the doors of a potential rapid pathogen detection era. 
For the empirical treatment of TB, a 4-drug regimen including isoniazid, rifampin, pyrazinamide, and ethambutol, was initiated. Erlich Ziehl-Neelsen stain (EZN), TB culture and PCR using Mycobacterium tuberculosis complex universal T4/T5 primers (9) for targeted pathogens were also negative for the peritoneal \&bronchoalveolar lavage samples. After six months with standard tuberculosis treatment, the patient fully recovered, and repeated abdominal CT revealed no infiltrative density changes or ascites.

The peritoneal fluid sample used for the routine tests was also used in this work after obtaining patient consent.

Patient-2: A 27-year-old female was presented with fever and chills, weakness and nausea. She had back pain for one week and was suffering from night sweats. She stated that she had a weight loss of about $4 \mathrm{~kg}$ in the last one month. The patient has a history of handling of stillbirth animal. She was treated for brucellosis ten months ago, but she did not have a clear knowledge about the type and duration of the drugs used for the treatment. On physical examination, her body temperature was $38.8^{\circ} \mathrm{C}$, and blood pressure was $120 / 70 \mathrm{mmHg}$, pulse rate was $92 / \mathrm{min}$. Her cardiac examination revealed normal heart sounds with no obvious extra sounds. The respiratory examination was normal. Minimal splenomegaly was observed but no hepatomegaly was found in the physical exam. In laboratory analysis, her complete blood count and biochemical parameters were as follows: WBC: $5.6 \times 103 /$

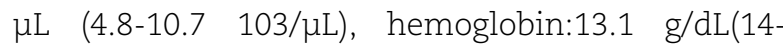

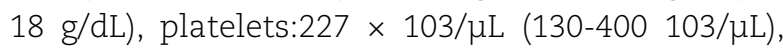
BUN:11.3 mg/dL(6-20 mg/dL), creatinine:0.71 mg/dL (0.7-1.2 mg/dL), AST:53 U/L (0-40 U/L), ALT:63 U/L (0-41 U/L), ALP:79 U/L(40-130 U/L), GGT:44 U/L(10$71 \mathrm{U} / \mathrm{L})$ and LDH:285 U/L(135-225 U/L). Her C-reactive protein level was $85 \mathrm{mg} / \mathrm{L}(0-6 \mathrm{mg} / \mathrm{L})$. Lumbar MRI was consistent with spondylodiscitis showing intense heterogeneous contrast enhancement at L4-L5 after contrast infusion. We performed serum tube agglutination test with Coombs and found titers to be $1 / 20$ and $1 / 40$, respectively. BacT/ Alert $\Theta$ 3D system (bioMérieux) and the standard laboratory procedures were able to reveal Brucella spp. after seven days. She received prolonged treatment with the regimen, including rifampin $(600 \mathrm{mg} / \mathrm{d}$, orally), doxycycline (200 mg/d, orally) and streptomycin (1 g/day, IM).

The blood sample taken from the patient for the routine test was used in this work with patient consent.

\section{Shotgun Metagenomic Sequencing}

Ten $\mathrm{mL}$ peritoneal fluid and $6 \mathrm{ml}$ blood samples (two tubes) were obtained from Patient-1 and $\mathrm{Pa}$ tient-2, respectively. These samples were kept in $+4^{\circ} \mathrm{C}$ refrigerator for at most 2 hours. They were transferred to Molecular Microbiology Laboratory of Genome and Stem Cell Center under cold chain conditions and processed immediately for the microbial DNA isolation. DNA isolation and human DNA depletion from the samples were performed using MolYsis ${ }^{\mathrm{TM}}$-Complete5 system (Molzym). The Qubit dsDNA high sensitivity Assay Kit with Qubit 2.0 Fluorometer (ThermoFisher) was used for the measurement of dsDNA concentration. 100ng dsDNA for each sample were fragmented using g-TUBEs (Covaris). Sequence-ready libraries were generated using the Nanopore Sequencing kit (SQK-MAP006) and Low input expansion kit (EXP-LWI001) according to the protocol. Briefly, 80 ng of extracted genomic DNA from each sample was fragmented with g-TUBE (app 8Kbp) at $6000 \mathrm{rpm}$ for $1 \mathrm{~min}$. DNA end-repair and dA-tailing were done using NEB Next Ultra II End Repair/dA-Tailing Module (New England Biolabs, NEB) and followed by purification step using Agencourt AMPure XP bead (Beckman Coulter). Sequencing adapter ligation was done with Blunt/TA Ligase Master Mix (NEB) followed by additional Dynabeads MyOne C1 Streptavidin (ThermoFisher) purification. Each sequence ready library was sequenced on a separate flow-cell (FLOMAP103). In order to conduct sequencing, a 72-h protocol was initiated using the MinION control software, MinKNOW.

\section{Data processing and analysis}

Read event data were base-called by the software Metrichor using workflow v2.39.3. A Hidden Markov Model-based Viterbi decoder was used for base calling procedure. In order to facilitate and investigate timely detection, the obtained fasta files were processed using the WIMP taxonomy analysis workflow 
with a period of 10 minutes as the sequencing continues. Python programming scripts automatically checking the results were used to report detection periodically. Confident detection of a pathogen is determined to be a total of $100 \mathrm{Kbp}$ unique sequence with an average Phred quality score of 10 . Here the unique sequence is defined as a read classified at the species level by the WIMP pipeline. Final classification generated reads to confirm the pathogens were done using Blastn (BLAST+ 2.8.1 using RefSeq (11) bacteria database) searches with parameters (word_size $=7$, match_score $=1$, mismatch_score $=-1$, existence_gap_cost $=2$, extension_gap_cost $=2$ ).

\section{RESULTS}

The 2D sequence reads were generated for each sample. In order to assess the rapid-diagnostic potential of real-time sequencing, the generated data was re-analyzed at time intervals of 10 minutes. On average, $9 \mathrm{Mbp}$ DNA was read in 10 minutes intervals per sample. For the Patient-1, the first Mycobacterium tuberculosis classification was done in 20 minutes, and the confident detection thresholds were achieved in 50 minutes time-point. For the Patient-2 the first Brucella melitensis read was detected in 30 minutes time-point and the confident detection thresholds was satisfied in 60 minutes timepoint. A detection was considered to be confident if the best hit achieves $>97 \%$ alignment identity. The total pathogen detection time -including microbial DNA isolation and library preparation- was calculated to be about 6 -hours after receiving samples. In our experiments, after confident detection is achieved, we had continued 72-hour sequencing protocol to confirm the detections. At the end of sequencing, the detected pathogens were confirmed at whole-genomic level with a total of overlapping $85 \mathrm{Mbp}$ Mycobacterium tuberculosis, and 37Mbp Brucella melitensis genomic reads generated. The genome coverages were calculated to be $\sim 19 \mathrm{X}$ and $\sim 11 \mathrm{X}$ for M. tuberculosis and B. melitensis respectively.

\section{DISCUSSION}

The paradigm to diagnose infectious diseases has been relying on the detection of infectious agents in the clinical samples. The traditional methodology for the detection and identification of the pathogens involves conventional microbiology (2). However, even with cultivable bacteria, conventional methods might fail to reveal an organism in patients with signs and symptoms consistent with an infectious disease (12). These approaches typically require a detection window starting from several days up to weeks. Taking these limitations into consideration, in order to detect the pathogen rapidly, we employed the novel real-time nanopore sequencing technology in the detection of infectious agents in two crucial cases where traditional microbiological methods failed to reveal the pathogens timely. For both cases, the entire process (including sample preparation, library construction, sequencing, data analysis, and issuing reports) took about 6-hours with the low capital costs and minimal equipment need (13). Thus, in comparison, both with the traditional clinical microbial diagnostic methods and relatively novel other molecular approaches, it shortens the total turnaround time and decreased the cost significantly.

Although rapid detection of viral pathogens using real-time nanopore sequencing technology has been an area of emerging interest recently (13-15), the related work on detecting bacterial infectious agents has been scarce. Exact procedures are mainly defined on amplicon sequencing (16), and whole metagenome sequencing-based screening appears to be experimental and case-based, including our report. However, the accumulating evidence implies that the use of real-time shotgun sequencing of metagenomes could be quite feasible for rapid pathogen detection. Similar to the current report, Votintseva et al. (17) utilized nanopore sequencing of respiratory samples, in order to detect $M$. tuberculosis in a culture free fashion. They reported that they were able to detect the agent in shorter than 12 hours. Nilgiriwala et al. (18) employed metagenomic sequencing of decontaminated sputum sediments gathered from two pulmonary tuberculosis patients based on nanopore sequencing technology. Both reports indicate correct detection of $\mathrm{M}$. tuberculosis, complying with our results. In another study, cerebrospinal fluid samples of B. melitensis have been subjected to metagenomic sequencing for rapid clinical detection using Illumina technology (19). However, a case study of detecting this pathogen from clinical specimens using real-time 
sequencing has not been reported yet, except for in silico pathogen detection simulations on synthetic data (20).

Although this novel approach brings invaluable advantages, still there are issues to be addressed and improvements to be made. Regarding experimental designs, further technological advancements that can handle low DNA inputs is needed to be developed, as this might be the case for most clinical applications. In a variety of clinical sample types, such as blood samples, the genomic content that is used for library construction after removing the human host cells appear to be very low, being even at the nanogram range, which might be a fundamental source of false-negative results. Therefore, simplified sample preparing procedures are required that can reduce the detection duration that is not requiring highly skilled and trained technical staff. In fact, library preparation appears to be the bottleneck of the current workflow, rather than real-time sequencing. Novel lab-on-a-chip automatized technologies such as Voltrax (21) are becoming deployable, and these advances have potential to standardize and speed-up the wet lab workflow, which could be invaluable for routine rapid pathogen detection of clinical specimens.

In addition to this, in the present work, we only employed nanopore sequencing to perform detection and identification of clinically relevant pathogens. However, both drug susceptibility and genotyping will be carried out in the future using the sequencing data generated for this report.

In summary, despite all advancements in molecular biotechnology, rapid pathogen detection and identification of infectious agents remains a challenging problem to be addressed that is essential to manage the therapy of infectious diseases. This becomes a crucial quest when vital treatment decisions must be made in a tight time frame, such as in the cases of sepsis in which a window of 6 hours is critical (22). Thus, rapid diagnostic procedures with minimal microbiological analysis requirements are needed. In this report, we showed that real-time nanopore sequencing technology is a plausible candidate to mitigate this problem.
Ethical Approval: Written consent was obtained from the patients. Peer-review: Externally peer-reviewed

Author Contributions: Concept - A.G, O.U.N.; Design - A.G., A.U.K., O.U.N., H.K; Supervision - A.G, O.U.N.; Materials - A.U.K, H.K.; Data Collection and/or Processing - A.U.K, H.K.; Analysis and/or Interpretation - A.G., A.U.K, H.K.; Literature Review - A.G.; Writer - A.G., A.U.K, O.U.N.; Critical Reviews - A.G., A.U.K, H.K, O.U.N.
Conflict of Interest: The authors have no conflict of interest to declare.

Financial Disclosure: This study was supported by The Scientific and Technological research council of Turkey (Grant Number: 116S083)

Sattement: This study was presented at the $27^{\text {th }}$ ECCMID Congress in 2017 as an oral-presentation.

\section{REFERENCES}

1 Lozano R, Naghavi M, Foreman K, Lim S, Shibuya K, Aboyans V, et al. Global and regional mortality from 235 causes of death for 20 age groups in 1990 and 2010: a systematic analysis for the Global Burden of Disease Study 2010. Lancet 2012; 380: 2095-2128.

2 Hasman H, Saputra D, Sicheritz-Ponten T, Lund O, Svendsen AA, Frimodt-Moller N, et al. Rapid whole-genome sequencing for detection and characterization of microorganisms directly from clinical samples. J Clin Microbiol 2014; 52: 139-146.

3 Ni P-X, Ding X, Zhang Y-X, Yao X, Sun R-X, Wang P, et al. Rapid detection and identification of infectious pathogens based on high-throughput sequencing. Chin Med J (Engl) 2015; 128: 877-883.

4 Neville SA, Lecordier A, Ziochos H, Chater MJ, Gosbell IB, Maley MW, et al. Utility of matrix-assisted laser desorption ionization-time of flight mass spectrometry following introduction for routine laboratory bacterial identification. J Clin Microbiol 2011; 49: 2980-4.

5 Wallet F, Nseir S, Baumann L, Herwegh S, Sendid B, Boulo M, et al. Preliminary clinical study using a multiplex real-time PCR test for the detection of bacterial and fungal DNA directly in blood. Clin Microbiol Infect 2010; 16: 774-9. 
6 Gu W, Miller S, Chiu CY. Clinical metagenomic next-generation sequencing for pathogen detection. Annu Rev Pathol-Mech Dis 2018, 14(1); 317-336.

7 Miller S, Chiu C. Metagenomic next-generation sequencing for pathogen detection and identification. Advanced Techniques in Diagnostic Microbiology 2018, 617-632.

8 Didelot X, Bowden R, Wilson DJ, Peto TE, Crook DW. Transform ing clinical microbiology with bacterial genome sequencing. Nat Rev Genet 2012; 13: 601-612.

9 Hellyer TJ, Desjardin LE, Assaf MK Bates JH, Cave MD, Eisenach KD. Specificity of IS6110-based amplification assays for Mycobacterium tuberculosis complex. J Clin Microbiol 1996; 34; 2843-2846.

10 Pruitt KD, Tatusova T, Maglott DR. NCBI reference sequences (RefSeq): a curated non-redundant sequence database of genomes, transcripts and proteins. Nucleic Acids Res 2006; 35: D61-D65.

11 Cheng J, Hu H, Kang Y, Chen W, Fang W, Wang K, et al. Identifi cation of pathogens in culture-negative infective endocarditis cases by metagenomic analysis. Ann Clin Microbiol Antimicrob 2018; 17: 43.

12 Judge K, Harris SR, Reuter S, Parkhill J, Peacock SJ. Early insights into the potential of the Oxford Nanopore MinION for the detection of antimicrobial resistance genes. J Antimicrob Chemother 2015; 70: 2775-2778.

13 Filloux D, Fernandez E, Loire E, Claude L, Galzi S, Candresse T, et al. Nanopore-based detection and characterization of yam viruses. Sci Rep 2018; 8: 17879.

14 Gallagher MD, Matejusova I, Nguyen L, Ruane NM, Falk K, Macqueen DJ. Nanopore sequencing for rapid diagnostics of salmonid RNA viruses. Sci Rep 2018; 8: 16307.

15 Xu Y, Lewandowski K, Lumley S, Pullan S, Vipond R, Carroll M, et al. Detection of viral pathogens with multiplex Nanopore MinION sequencing: be careful with cross-talk. Front Microbiol 2018; 9: 2225.

16 Mitsuhashi S, Kryukov K, Nakagawa S, Takeuchi JS, Shiraishi Y, Asano K, et al. A portable system for rapid bacterial composition analysis using a nanopore-based sequencer and laptop computer. Sci Rep 2017; 7: 5657.

17 Votintseva AA, Bradley P, Pankhurst L, del Ojo Elias C, Loose M, Nilgiriwala $\mathrm{K}$, et al. Same-day diagnostic and surveillance data for tuberculosis via whole-genome sequencing of direct respiratory samples. J Clin Microbiol 2017; 55: 1285-1298.

18 Nilgiriwala KS, Pankhurst L, Vaughan A, Iqbal Z, Crook D, Chatterjee A, et al. Towards point-of-care diagnosis of pulmonary tuberculosis and drug susceptibility testing by whole genome sequencing of dna isolated from sputum. Can J Biotech 2017; 1: 267.

19 Bachmann NL, Rockett RJ, Timms VJ, Sintchenko V. Advances in clinical sample preparation for identification and characterization of bacterial pathogens using metagenomics. Front Public Health 2018; 6: 363.

20 Pearman WS, Freed NE, Silander OK. The advantages and disadvantages of short-and long-read metagenomics to infer bacterial and eukaryotic community composition. BioRxiv 2019; 650788.

21 Oxford Nanopore Technologies. VolTRAX: a rapid, programmable, portable, disposable device for sample and library preparation. Available from: URL: https://nanoporetech.com/ publications/voltrax-rapid-programmable-portable-disposable-device-sample-and-library-preparation.

22 Kumar A, Roberts D, Wood KE, Light B, Parillo JE, Sharma S, et al. Duration of hypotension before initiation of effective antimicrobial therapy is the critical determination of survival in human septic shock. Crit Care Med 2006; 34: 1589-1596. 